\title{
The hypothesis of elementary dimensions' role in the creation of the source of elementary particles
}

\author{
Shivan Sirdy \\ Science, Kirkuk University, Kirkuk, 36000, Iraq
}

Keywords: Early universe, Elementary dimensions, Gravitational collapse, High energy physics, The Quark Gluon-Plasma, Inflation of the universe, gravitational lensing

\begin{abstract}
Assuming that there is a chance that the big bang theory is wrong and that concept of time and space didn't start with the big bang. However, it's undeniable per observations that the universe is in expansion. Now before the creation of particles, there was a space devoid of matter and any sorts of radiations (electromagnetic radiations, heat radiations...), meaning in this space the absolute zero temperature is reached naturally. We will call this space an absolute void. It has four dimensions (the three dimension of space $\mathrm{x}$ axis, $\mathrm{y}$ axis, and $\mathrm{z}$ axis and the force). The fourth dimension (the force) is the factor of change among the four. Those four are elementary dimensions. The hypothesis is that when the space dimensions increases the outward force subsequently increases as well and the space increases its instability and losses its equilibrium. Now this region of space has only outward force, when the outward force exceeds a certain limit, the system will be at its highest level of instability. To create equilibrium in the system the outward force collapses inward. The void inside the collapsed force will act as void in a confined system. Meaning now, the void in a confined system is the source of the elementary particles. Now the created source is being managed by internal force and external force. The two forces will affect the source until equilibrium is reached between the internal and external forces of source and the entities in its external force field effect. The source is a high energy entity. As before the creation of particles, there was infinity space of absolute void. Therefore, unlimited numbers of Sources were created.

The Quark Gluon-Plasma, the Inflation of the universe, the gravitational collapse and gravitational lensing are all proofs for the elementary dimensions theory. Each of these are written about in this paper, as there are not enough space in the abstract to mention all of them.
\end{abstract}

Email address: Sheevan91@gmail.com (Shivan Sirdy) 


\section{Table of contents}

Table of contents

Introduction to Void and the Elementary dimensions ................................................................ 2

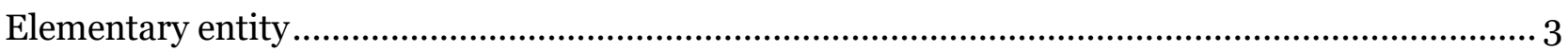

The hypothesis of creation of the source of elementary particles through the elementary

dimensions

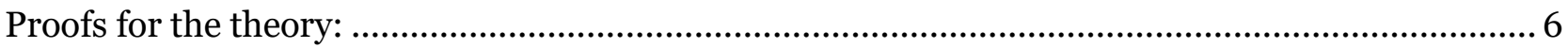

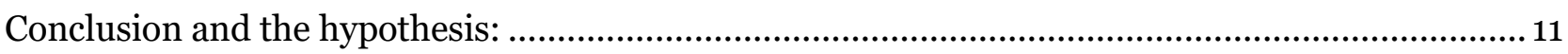

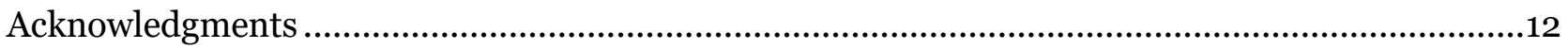

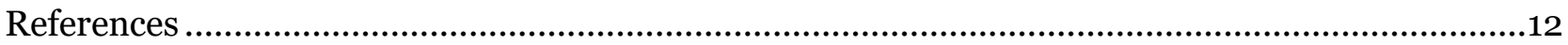

\section{Introduction to Void and the Elementary dimensions}

Assuming that there is a chance that the big bang theory is wrong and that concept of time and space didn't start with the big bang. However, it's undeniable per observations that the universe is in expansion. It might be difficult to adopt such assumption, as we were as physics passionate raised on the idea that the universe start with the big bang. Now that idea aside, before the creation of particles, we can agree that there was a space devoid of matter and any sorts of radiations (electromagnetic radiations, heat radiations...), meaning in this space the absolute zero temperature is reached naturally. We will call this kind of space absolute void and the space devoid matter only as a void, where void can contain radiations such as heat, electromagnetic radiations.

For starter we can say that void and absolute void has three dimensions (the three dimension of space $\mathrm{x}$ axis, $\mathrm{y}$ axis, and $\mathrm{z}$ axis). If void only has those three dimensions, it means that change will not occur and the void will stay in the existence as the only entity. However, we must have a fourth dimension that will lead to creation of matter or particles. So the question is what is the fourth dimension? Is time the fourth dimension?

To answer the above question, we will need to know what time actually is. Analyzing time thoroughly we will see that the concept of time is something hypothetical, expresses changes that occurs in a certain event compared to number of changes in a constant change rate event. Meaning the concept of time has been made by human being to organize their lives and their histories. The time doesn't really exist, however, as living beings we can sense the effects of time. Therefore, the answer is no. Time is not the fourth dimension. Time represents a factor of change. So the factor of change is the fourth dimension.

So what is the factor of change in void?

To answer this question, we will need to study the properties of void and absolute void from experiment. However, it's very difficult to create a volume of absolute void, as it's extremely difficult to extract all particles and radiations from a specific volume of space. Therefore, in the experiment we will create void only (a space devoid of particles). Still it's difficult to create void totally devoid of particles, however through the experiment, at least we will be able to identify the fourth dimension (the factor of change).

In the figure 1 below shows the void creation through pulling the grey mass downward: 

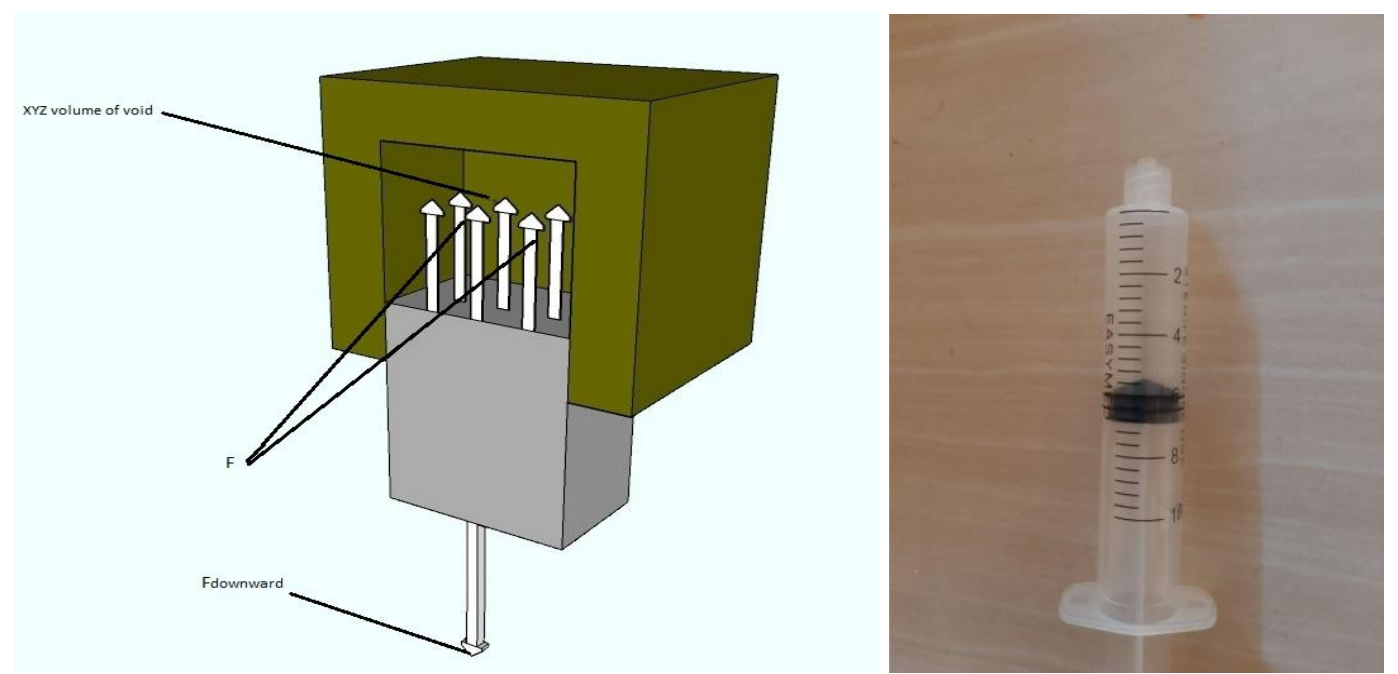

Figure 1 (void creation experiment through using a closed head syringe)

Due to lack of resources, a $10 \mathrm{ml}$ syringe was used to identify the elementary dimensions of the void. Where, the syringe's head was closed and the bottom part was pulled using weights to create the void in the syringe

Through classic physics, summation of forces on the vertical axes equal zero $\Sigma \mathrm{Fy}=0, \mathrm{~F}+\mathrm{Fdownward}=\mathrm{O}$

Fdownward is the force needed to pull the matter downward to create $1 \mathrm{~m}^{3}$ of void and $\mathrm{F}$ is the force sourced from the void to return the matter to remove the void's creation.

F- Fdownward $=\mathrm{O} \rightarrow \mathrm{F}=$ Fdownward

Therefore as shown in the experiment, that void has a property which is the force $\mathrm{F}$ resisting to create the void.

Therefore, the force $\mathrm{F}$ is the factor of change. Meaning $\mathrm{F}$ is the fourth dimension.

As already mentioned, the force identified in this experiment is for void. The force developed in an absolute void space is different, most probably, the force in absolute void is way stronger.

\section{Elementary entity}

Void or absolute void is an elementary entity in the existence and it is the source of everything else.

It has four dimensions $\mathrm{X}$ axis, $\mathrm{Y}$ axis, $\mathrm{Z}$ axis and the force.

The absolute void may exists as free entity (the spaces that particles have not been created yet and radiations haven't reach them yet or universe before creation particles). While, void may exists in the existence as a part of the particles. Taking any simple atom as an example, where around more than $96 \%$ of the volume of any atom is occupied by void. Below, I will show the force pattern direction of a void space in a confined system and an open system. 
1. Void in a confined system:

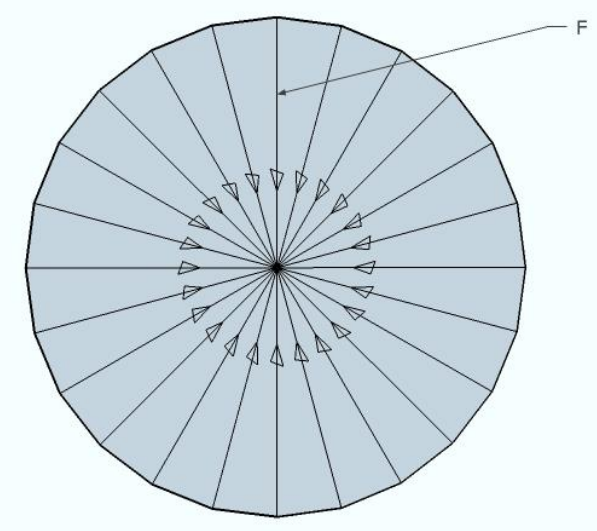

Figure 2 (The forces sourced from void in a confined system)

As mentioned, the attached figure shows the forces sourced from void in a confined system (similar to experiment conducted as in figure 1). The force tries to eliminate the void's creation through trying to crush the parameter of the surroundings toward the center of void. Meaning the directions of $\mathrm{F}$ will be toward the center of void.

2. Void in an open system:

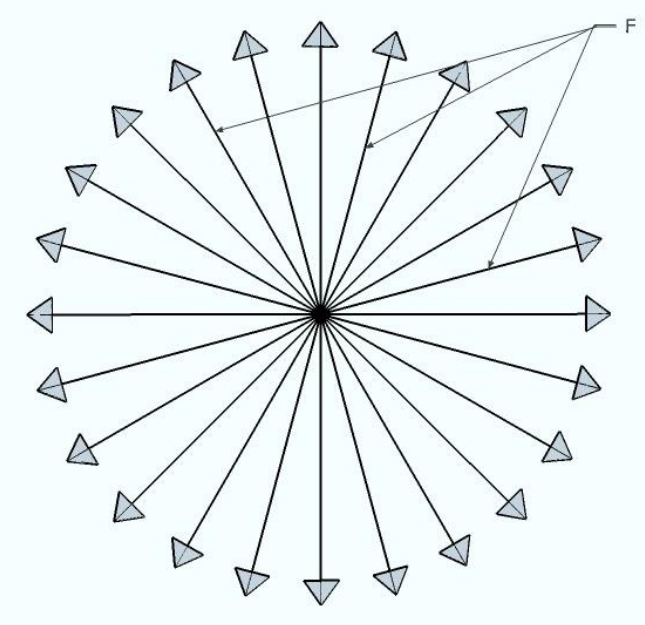

Figure 3 (The forces sourced from void in an open system)

Obviously, void in an open system, mean the void's existence in a free form. Where the void is not surrounded by particles (just like the void's existence before the creation of particles). Taking any point of void, we will see that void's force is outward, just as depicted in the figure 3 . 
As well taking any point of void in a confined system will be acting as void in an open system, meaning the directions of forces are outwards (like the below figure).
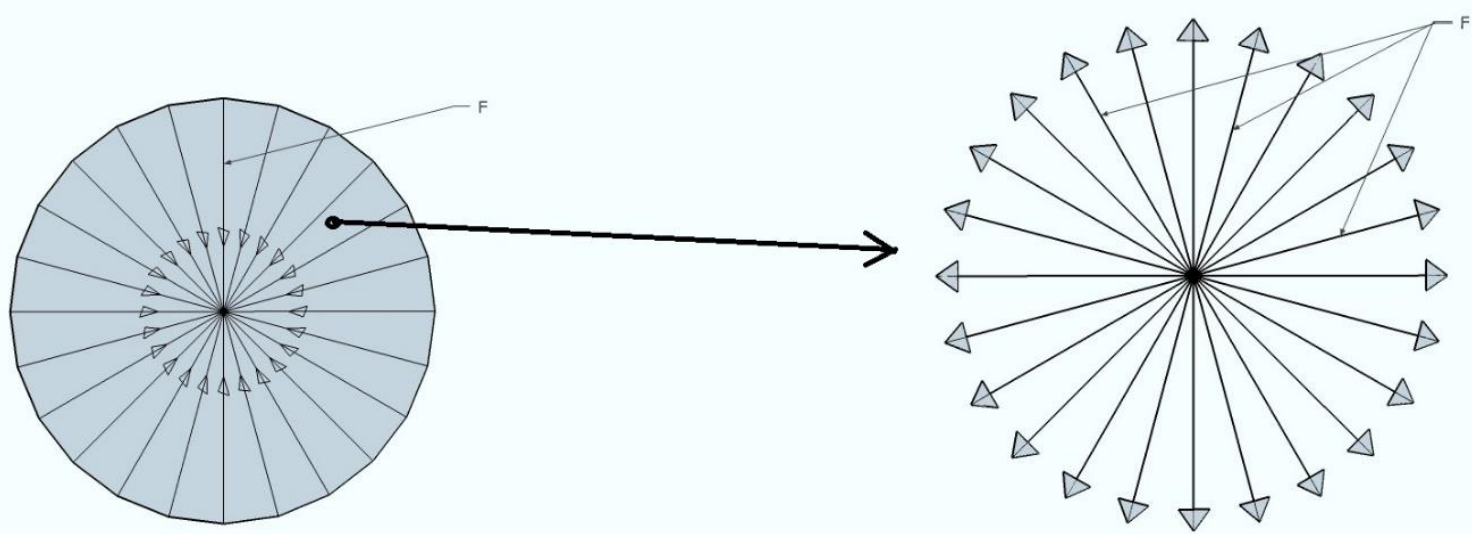

Figure 4 (any point of void in a confined system will be acting as void in an open system)

\section{The hypothesis of creation of the source of elementary particles through the elementary dimensions}

As mentioned above that we have four elementary dimensions and its fourth dimension is the force the factor of change. $\mathrm{XYZ}$ represents the volume of void and when the volume of void increases the outward force subsequently increases as well and the space increases its instability and losses its equilibrium. Now this region of space has only outward force, when the outward force exceeds a certain limit, the system will be at its highest level of instability. To create equilibrium in the system the outward force collapses inward.

(As mentioned in the below figure 5)
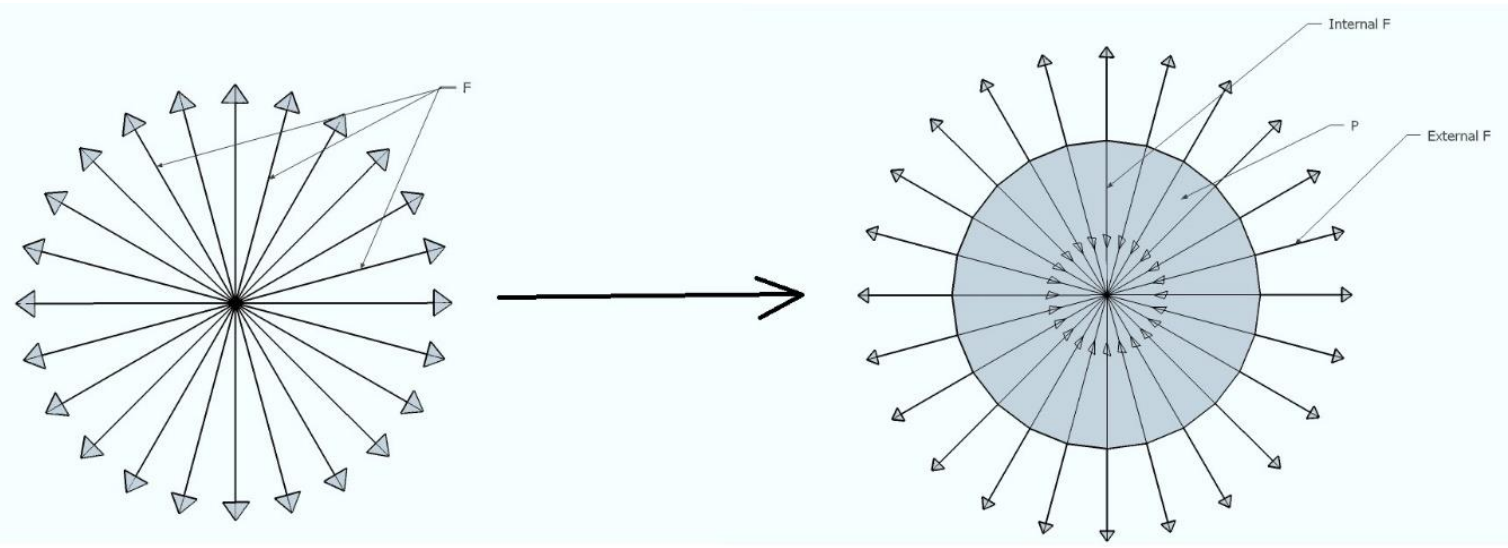

Figure 5 (transformation of void in an open system to void in a confined system or the birth of elementary particle)

Now the void has transformed from void in an open system to void in a confined system. The void in a confined system is the source of the elementary particles. The Source of elementary particles was created to absorb the surplus force and to decrease the level of 
instability in the system and to achieve equilibrium in space. We will call the source of elementary particles just the Source for simplification.

At this stage, we have the source. Now it is being managed by two forces:

a. Internal force Fi: this force is from the void inside the particle, just like void in a confined system, its direction is toward the centre.

b. External force Fe: this force is from the void outside the born particle.

The Fi makes the Source shrinking or collapsing, while the Fe is the resisting force. Meaning the Source collapses till equilibrium is achieved between the internal force and the external forces.

The source just like the elementary particles is a high energy entity.

As before the creation of particles, there was infinity space of absolute void. Therefore, unlimited numbers of Sources were created. The interactions and collisions among the created Sources led to creating the elementary particles and the process continued to form the universe we are in. Unfortunately, for now it will be mere assumptions writing in precision what happened after the Source was created.

\section{Proofs for the theory:}

For simplification we can call the mentioned theory above the elementary dimensions theory EDs:

There are many phenomena that proofs the theory, however here we will go through four:

\section{Quark-gluon plasma or QGP}

Before jumping to QGP, we will need to understand what the Hagedorn temperature (Gaździcki \& Gorenstein, . (2016),) is, or $\mathrm{T}_{\mathrm{h}}$, is the temperature in theoretical physics where hadronic matter (i.e. ordinary matter) is no longer stable, and must either "evaporate" or convert into quark matter; as such, it can be thought of as the "boiling point" of hadronic matter.

Now QGP (Bhalerao, (2014).) is an interacting localized assembly of quarks and gluons at thermal (kinetic) and close to chemical abundance equilibrium. The word plasma signals that free color charges are allowed. Since the temperature is above the Hagedorn temperature-and thus above the scale of light up and down-quark mass-the pressure exhibits the relativistic Stefan-Boltzmann format governed by fourth power of temperature and many practically mass free quark and gluon constituents. We can say that QGP emerges to be the new phase of strongly interacting matter which manifests its physical properties in terms of nearly free dynamics of practically massless gluons and quarks. Both quarks and gluons, must be present in conditions near chemical (yield) equilibrium with their colour charge open for a new state of matter to be referred to as QGP.

Now as a process starting from: 
1. Room degree temperature for a regular atoms as an example, the structure of the atoms would be at a solid state.

2. Increasing the temperature will increase the level of freedom in the atoms, which will change the structure to a liquid state.

3. Increasing the temperature more, the level of freedom increases, changing the structure to a gas state.

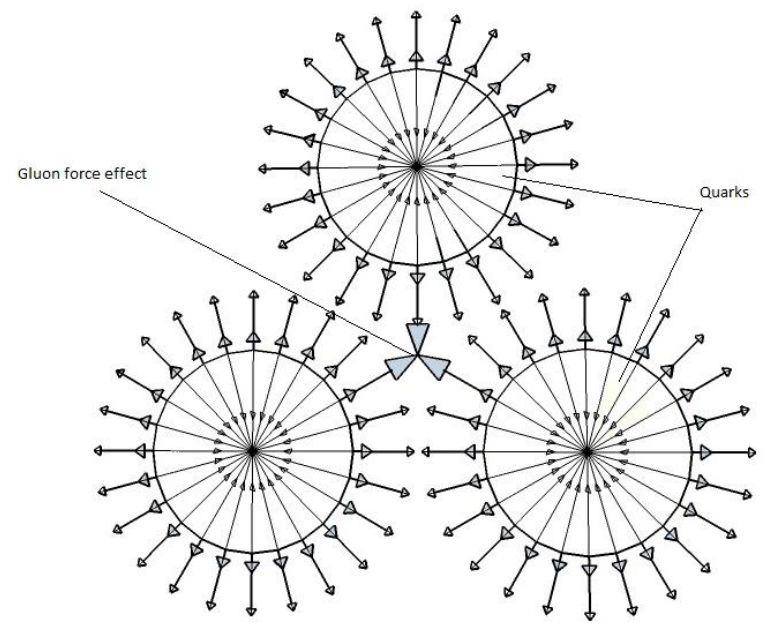

Figure 6 (the hadron before reaching the QGP state, the gluon force is sill active)

4. Eventually increasing the temperature excessively will lead to the QGP state.

Now obviously, the above statement is different in what is mentioned in the elementary dimensions theory EDs. Where EDs says that before the creation of particles, the universe was in an absolute zero temperature.

Indeed, at the absolute zero temperature, the opposite of QGP occurs.

When the temperature decreases to the absolute zero, the process will go in reverse, where the level of freedom decreases to the solid state and then the level of freedom decreases to the state where all the components of the atom would be merged together. Meaning, the fermions and all the types of bosons will be in the same place. Meaning, what's known as singularity (Shapiro \& Teukolsky, (1991)) will be achieved.

Definition of singularity is a location where the quantities that are used to measure the gravitational field become infinite in a way that does not depend on the coordinate system. In other words, it is a point in which all physical laws are indistinguishable from one another, where space and time are no longer interrelated realities, but merge indistinguishably and cease to have any independent meaning.

According to the EDs theory, the singularity is a state where the absolute zero temperature is reached and all the components of particle (fermions and bosons) are in the same location. At the singularity state, the particle shows its maximum mass level.

Meaning the created Source of elementary particles from the elementary dimensions is at the singularity state and at its maximum mass level in the very small scale of space dimensions (XYZ). 
2. Inflation of the universe (Overbye, 20 February 2017) (Whiting, 2004)

As mentioned at the first proof that the Source is at singularity (at absolute zero temperature) and at a very small scale of the dimensions of space (XYZ), when the temperature increases the system will no longer stay at singularity state and the space dimensions consequently increases, leading to the expansion of universe at every directions. The source for the increasing temperature could be the interactions and collisions among the created Sources in the early universe (as mentioned earlier that before the creation of particles, there was infinity space of absolute void. Therefore, unlimited numbers of Sources were created).

Meaning, the universe will keep expanding as far as there is a source of heat radiations that makes the temperature increasing.

Concluding that, when the universe consumes all the sources of heat radiations, the universe will crunch.

3. The gravitational collapse: we can say that the gravitational collapse is similar to the process in which void in an open system becomes a void in a confined system or change of void's force from outward to inward. The force (the fourth dimension) mentioned in the illustrations above is the gravitational force.

The gravitational collapse is happening in the universe, as there are observation proofs in its regard. However, just to emphasize I don't mean that the observed gravitational collapse product (only the black hole) is the Source itself. While, the process that leads to its creation is the same as the Source creation.

Which more proves that they are the similar, the fact that the black hole is also high energy entity just like the Source. The explanation below shows the similarity:

a. The Source in equilibrium state:

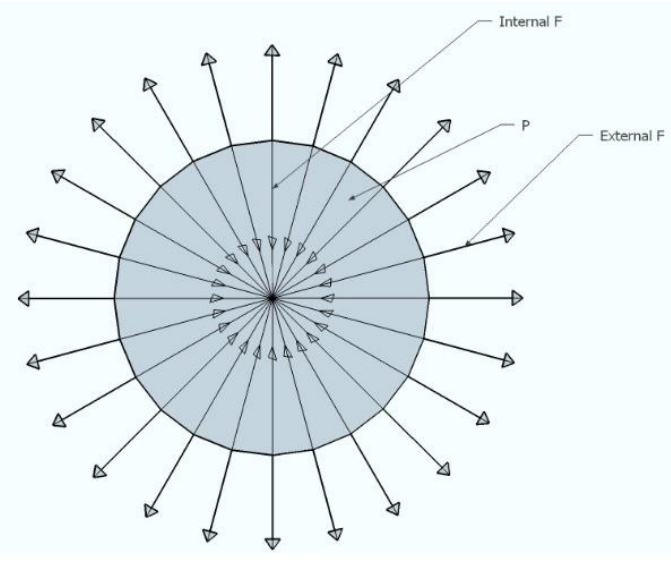

Figure 7 (the Source in a state of equilibrium Fi=Fe)

As already mentioned the internal force makes the Source shrinking or collapsing, while the external force is the resisting force. The Source collapses till equilibrium is achieved between the internal and the external forces. 
The equilibrium of the source is also effected by the other entities (other Sources, stars, planets...) in its external force field (see the below figure). Since those entities also have their own external and internal forces. Now the equilibrium will be among the external and internal forces for all the entities including the Source.
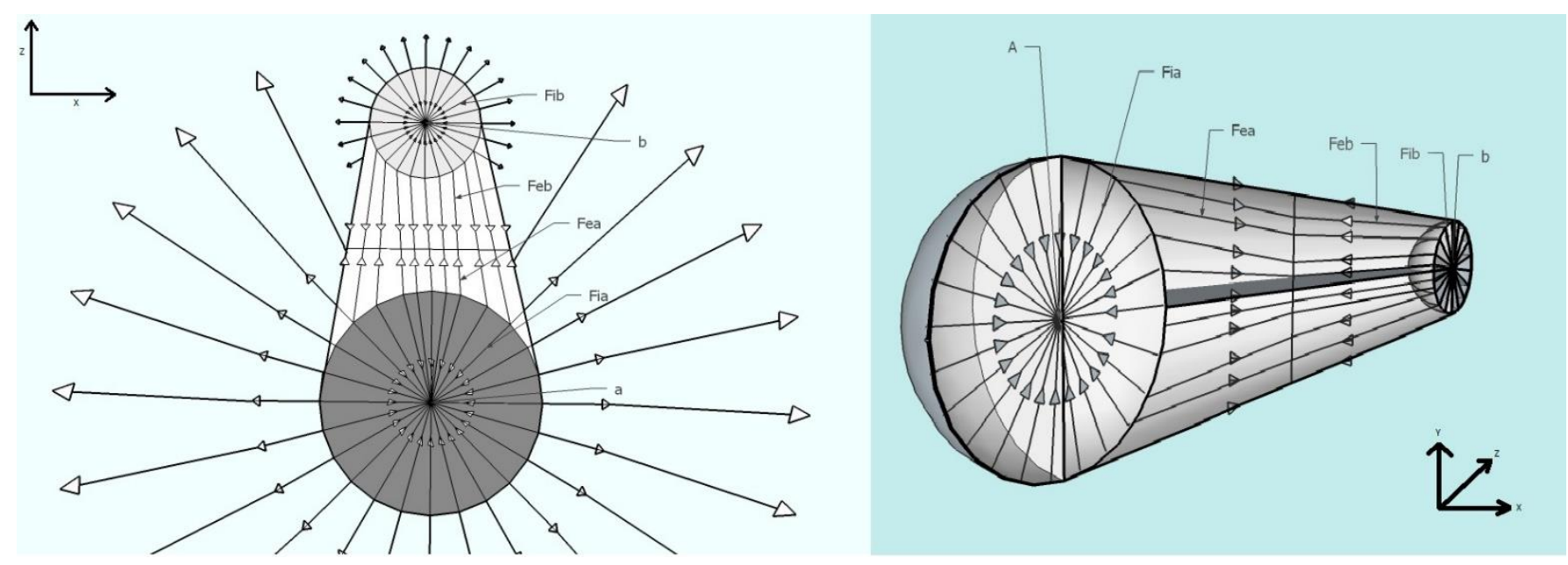

Figure 8 (the Source in a state of equilibrium with all the entities in its field of impact Fi total =Fe total)

b. The black hole in equilibrium state:

Before the star dies, the space effected by the star is in equilibrium between the external and the internal forces for all the entities in the field. The stars and any other forms of particles clusters have internal and external forces, however, the amount is much less considering that they are not high energy entities.

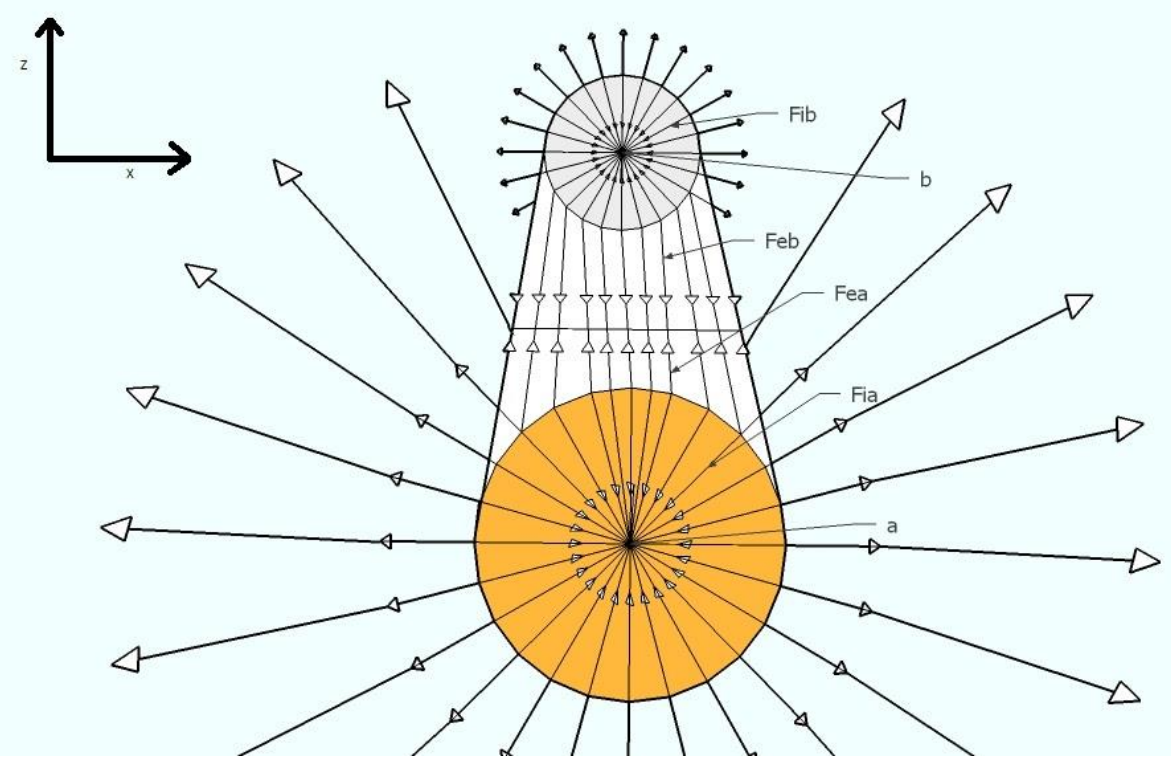

Figure 9 (a Star in a state of equilibrium with all the entities in its field of impact Fi total=Fe total (the figure is not drawn on scale, it's merely for illustration)) 
According to Einstein's theory, for large stars, above the Landau-Oppenheimer-Volkoff limit, also known as the Tolman-Oppenheimer-Volkoff limit (around the twice mass of our Sun) no known form of cold matter can provide the force needed to oppose gravity in a new equilibrium. Hence, the collapse continues with nothing to stop it.

Once a body collapses to within its Schwarzschild radius it forms a black hole, meaning a space-time region from which not even light can escape. It follows from general relativity and the theorem of Roger Penrose (Penrose, 1965-01-18) that the subsequent formation of some kind of singularity is inevitable. Nevertheless, according to Penrose's cosmic censorship hypothesis, the singularity will be confined within the event horizon bounding the black hole, so the space-time region outside will still have a well behaved geometry, with strong but finite curvature, that is expected (Carter, 197102-08) to evolve towards a rather simple form describable by the historic Schwarzschild metric in the spherical limit and by the more recently discovered Kerr metric if angular momentum is present.

Therefore, the black hole is formed to restore the equilibrium to the system.

Depending on the elementary dimensions analysis, the space where the big star is dominant. The star has the largest amount of the internal force among all the entities, meaning, equilibrium of that region of space is mostly dependent on the star's internal force. When the star dies the system losses its equilibrium.

A star is not a high energy entity regardless of how big it is. Therefore, a much smaller high energy entity is needed to take the star's place in the equilibrium process.

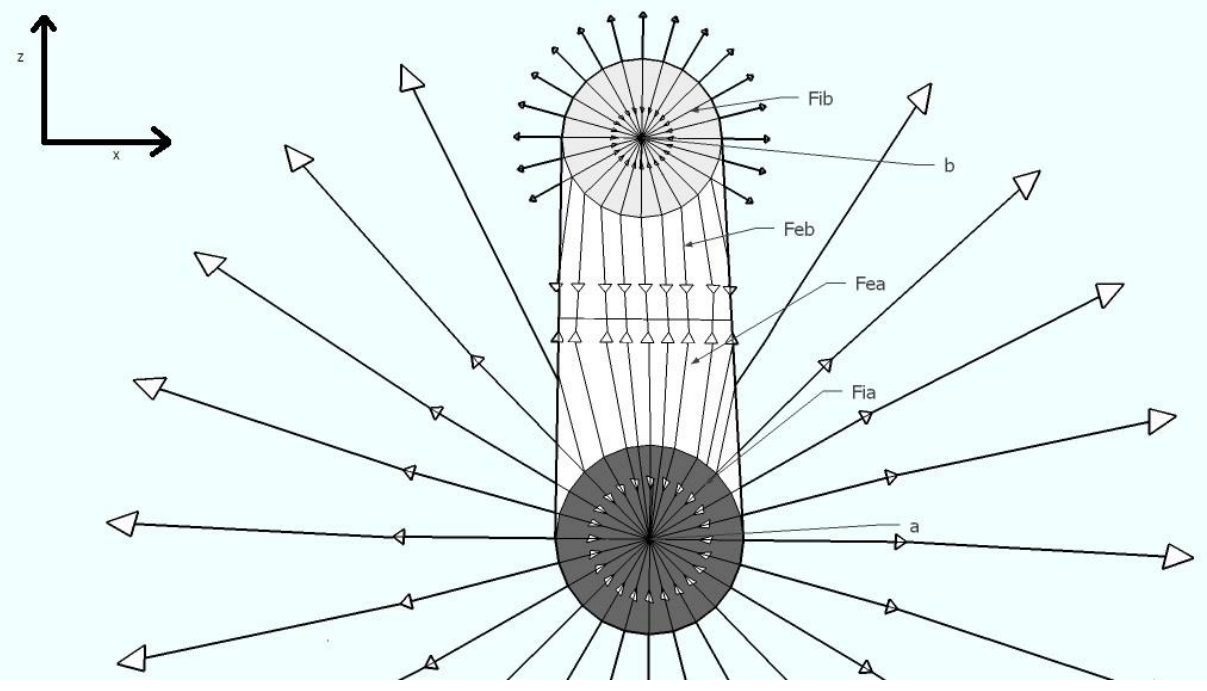

Figure 10 (the formed black hole in a state of equilibrium with all the entities in the dead star's field of impact Fi total =Fe total (the figure is not drawn on scale, it's merely for illustration)

We can say that the source is formed to create equilibrium in the space, as before the Sources creation, there was only the external force. The sources forms at size as big as solar sizes and collapses till the equilibrium is achieved with the external force in the space and the black hole is formed to restore and fill the gap in the equilibrium in the space, developed due to the star's death. 


\section{The gravitational lensing (Einstein, 1936):}

It is a distribution of matter (such as a cluster of galaxies) between a distant light source and an observer that is capable of bending the light from the source as the light travels towards the observer (Sauer, 2008).

The gravitational lensing fits perfectly in the EDs theory, the figures below shows the reasons (the figures aren't drawn on scale, they are merely for illustrations:
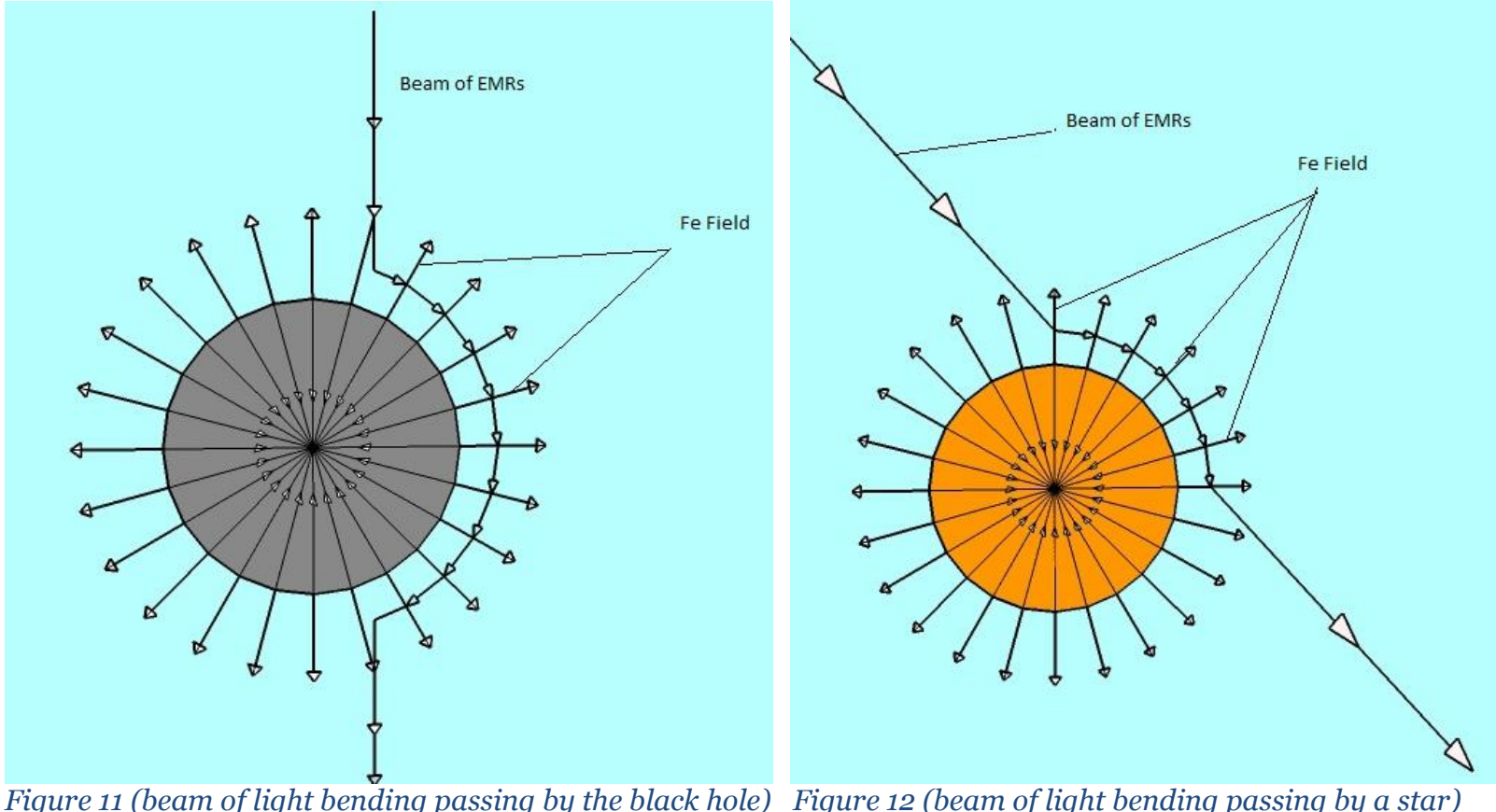

Figure 12 (beam of light bending passing by a star)

When the beam of light approaches the black hole or the star, the light beam enters the external force field effect of the system. It's mentioned earlier that the Fe is an outward force. Whereby, the Fe prevents the light beam from falling to inside the system, which causes what's known as the gravitational lensing.

5. There must be a source for the creation of particles, particles can't just explode out of space and create the universe.

The above reason may sound lazy. However, the particles have to be created from the void and its dimensions, since void is the predecessor of particles.

\section{Conclusion and the hypothesis:}

Conclusion: Absolute void has four elementary dimensions, three of them are space dimensions and the fourth is the factor of change among them.

The hypothesis: There is a limit in the level of instability of absolute void at absolute zero temperature, where the external force is too huge. When the external force exceed that limit, the force collapses inward, creating what we can call as the source of elementary particles. The Source is governed by two forces, the external force and the internal. The internal force makes the Source collapsing, while the external force is the resistant force. The source will be in state of equilibrium, when the external force equals the internal force. The source is a 
high energy entity and the source exists at absolute zero temperature, meaning all the components of the particle are in the same location (singularity according to EDs theory).

There are uncountable proofs for the theory, the above written 5 proofs are merely 5 phenomena that came to my mind.

\section{Acknowledgments}

I would like to express my special thanks of gratitude to the University of Kirkuk for giving me the faith and the time required to complete the paper.

\section{References}

1. Gaździcki, Marek; Gorenstein, Mark I. (2016), Rafelski, Johann (ed.), "Hagedorn's Hadron Mass Spectrum and the Onset of Deconfinement", Melting Hadrons, Boiling Quarks - From Hagedorn Temperature to Ultra-Relativistic Heavy-Ion Collisions at CERN, Springer International Publishing, pp. 87-92, doi:10.1007/978-3-319-17545-4_11, ISBN 978-3-31917544-7, retrieved 2020-03-30

2. Bhalerao, Rajeev S. (2014). "Relativistic heavy-ion collisions". In Mulders, M.; Kawagoe, K. (eds.). 1st Asia-Europe-Pacific School of High-Energy Physics. CERN Yellow Reports: School Proceedings. CERN-2014-001 ; KEK-Proceedings-2013-8. Geneva: CERN. pp. 219-239. doi:10.5170/CERN-2014-001. ISBN 9789290833994. OCLC 801745660.

3. Shapiro, Stuart L.; Teukolsky, Saul A. (1991). "Formation of naked singularities: The violation of cosmic censorship" Physical Review Letters. 66 (8): 994997. Bibcode:1991PhRvL..66..994S. doi:10.1103/PhysRevLett.66.994. PMID 10043968.

4. Overbye, Dennis (20 February 2017). "Cosmos Controversy: The Universe Is Expanding, but How Fast?". The New York Times. Retrieved 21 February 2017.

5. Alan B. Whiting (2004). "The Expansion of Space: Free Particle Motion and the Cosmological Redshift". The Observatory. 124: 174. arXiv:astro-ph/0404095. Bibcode:2004Obs...124..174W.

6. Einstein, Albert (1936). "Lens-Like Action of a Star by the Deviation of Light in the Gravitational Field". $\quad$ Science. 84 (2188): 506-507. Bibcode:1936Sci....84..506E. doi:10.1126/science.84.2188.506. PMID 17769014.

7. Tilman Sauer (2008). "Nova Geminorum 1912 and the Origin of the Idea of Gravitational Lensing". Archive for History of Exact Sciences. 62 (1): 122. arXiv:0704-0963. doi:10.1007/s00407-007-0008-4.

8. ^ Penrose, Roger (1965-01-18). "Gravitational Collapse and Space-Time Singularities". Physical Review Letters. American Physical Society (APS). 14 (3): 57-59. doi:10.1103/physrevlett.14.57. ISSN 0031-9007.

9. ^ Carter, B. (1971-02-08). "Axisymmetric Black Hole Has Only Two Degrees of Freedom". Physical Review Letters. American Physical Society (APS). 26 (6): 331-333. doi:10.1103/physrevlett.26.331. ISSN 0031-9007. 